\title{
Taylor Coefficients of the Conformal Map for the Exterior of the Reciprocal of the Multibrot Set
}

\author{
Hirokazu SHIMAUCHI* \\ Policy Data Lab, The Tokyo Foundation for Policy Research, Tokyo 106-6234, Japan
}

\begin{abstract}
In this paper we investigate normalized conformal mappings of the exterior of the reciprocal of the Multibrot set and analyze the growth of the denominator of the coefficients. Our inequality improves Ewing and Schober's result which was presented in [6]. We use the coefficient formula of [13]. The straightforward adaptation of the proof in this paper slightly improves the main theorem of [12].
\end{abstract}

KEYWORDS: Mandelbrot set, Multibrot set, conformal mapping, complex dynamics

\section{Introduction}

Let $\mathbb{Z}$ be the set of all integers, $\mathbb{N}_{0}$ the set of all non-negative integers, $\mathbb{N}$ the set of positive integers, $\mathbb{C}$ the complex plane, $\mathbb{D}$ the open unit disk, $\overline{\mathbb{D}}$ the closed unit disk and $P: \mathbb{C} \rightarrow \mathbb{C}$ a non-linear polynomial. Let $p$ be a prime number and $x$ any non-zero rational number. Then there exists a unique integer $v$ such that $x=p^{v} r / q$ with integers $r$ and $q$ that are not divisible by $p$. We define the $p$-adic valuation $v_{p}: \mathbb{Q} \rightarrow \mathbb{Z}$ as follows:

$$
v_{p}(x)= \begin{cases}v & \text { for } x \in \mathbb{Q} \backslash\{0\}, \\ +\infty & \text { for } x=0 .\end{cases}
$$

For any real number $x$, we set the floor function $\lfloor x\rfloor:=\max \{m \in \mathbb{Z}: m \leq x\}$.

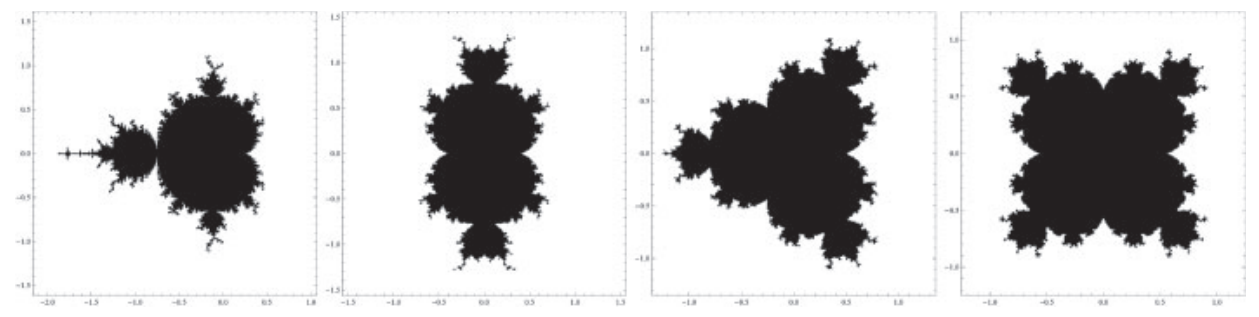

Fig. 1. $\mathcal{M}_{2}, \mathcal{M}_{3}, \mathcal{M}_{4}, \mathcal{M}_{5}$.

The $n$-th iteration of the polynomial $P^{\circ n}$ is defined inductively by $P^{\circ(n+1)}=P \circ P^{\circ n}$ with $P^{\circ 0}(z)=z$. The Multibrot set $\mathcal{M}_{d}$ of degree $d$ is the set of all parameters $c \in \mathbb{C}$ for which the Julia set of $P_{d, c}(z):=z^{d}+c$ is connected, where $d \geq 2$ is a fixed integer (see Fig. 1 for $d=2,3,4,5$ ). It is known that $\mathcal{M}_{d}$ is compact and connected. There is an important conjecture which states that the Mandelbrot set $\mathcal{M}_{2}$ is locally connected. This would imply the density conjecture of hyperbolic dynamics in the quadratic family (see [2]). Douady and Hubbard demonstrated connectedness of $\mathcal{M}_{2}$ by constructing a conformal isomorphism $\Phi: \widehat{\mathbb{C}} \backslash \mathcal{M}_{2} \rightarrow \widehat{\mathbb{C}} \backslash \overline{\mathbb{D}}$ that satisfies $\lim _{z \rightarrow \infty} \Phi(z) / z=1$. If the inverse map $\Phi^{-1}(z)=: \Psi(z)$ extends continuously to the unit circle, then $\mathcal{M}_{2}$ is locally connected, according to Carathéodory's continuity theorem. It should be noted that the convergence of the Laurent series $\Phi^{-1}(z)=z+\sum_{m=0}^{\infty} b_{m} z^{-m}$ on the unit circle implies that $\mathcal{M}_{2}$ is locally connected. Jungreis presented a method to compute the coefficients $b_{m}$ of $\Psi(z)$ in [8]. Several detailed studies of $b_{m}$ are given in $[1,4,5,10]$. An analysis of the dynamics of $P_{d, c}(z):=z^{d}+c$ with an integer $d \geq 2$ is presented in [15]. Constructing the canonical map $\Psi_{d}(z)=z+\sum_{m=0}^{\infty} b_{d, m} z^{-m}$ of $\widehat{\mathbb{C}} \backslash \mathcal{M}_{d}$, Yamashita [15] analyzed the coefficients $b_{d, m}$. The function $f_{d}(z):=1 / \Psi_{d}(1 / z)$ is the normalized conformal map $f_{d}$ from $\mathbb{D}$ to $\mathcal{R}_{d}:=\mathbb{C} \backslash\left\{1 / z: z \in \mathcal{M}_{d}\right\}$ which fixes the origin and satisfies $f_{d}^{\prime}(0)>0$. If $f_{d}$ has a continuous extension to the boundary, the Multibrot set is locally connected. The conformal map $f_{d}(z): \mathbb{D} \rightarrow \mathcal{R}_{d}$ has the Taylor expansion of the form $f_{d}(z)=z+a_{d, 1} z+a_{d, 2} z^{2}+a_{d, 3} z^{3}+\cdots($ see $[6,13])$. Ewing and Schober studied the coefficients $a_{2, m}$ in [6]. They 
gave some coefficients formulas and estimate for the growth of denominator, and presented that infinitely many coefficients are 0 . Also infinitely many non-zero terms are determined. In $[13,14]$, we investigated properties of the coefficients $a_{d, m}$ of the conformal map $f_{d}(z)=z+\sum_{m=2}^{\infty} a_{d, m} z^{m}$ of the exterior of $\mathcal{R}_{d}:=\left\{1 / z: z \in \mathcal{M}_{d}\right\}$. Some values of $a_{d, m}$ are presented in Tables $1,2,3,4$ for $d=2,3,4,6$ respectively.

In particular, $a_{d, m}$ is $d$-adic rational, i.e. there exist $k \in \mathbb{Z}$ and $n \in \mathbb{N}_{0}$ such that $a_{d, m}=k / d^{n}$ (see [13, Lemma 4]). Ewing-Schober [6] studied the coefficients of $f_{2}$ and obtained an inequality for the growth of the denominator, which can be presented in the following way.

Theorem 1.1 ([6, Theorem 2]). For $m \geq 2$, the inequality

$$
-v_{2}\left(a_{2, m}\right) \leq 2 m-3
$$

holds. Equality holds if $m=2^{k}+1$ for some $k \in \mathbb{N}$.

In this note, we improve this inequality in a general way. In particular, our result is as follows when $d=2$.

Corollary 1.2 (Corollary of the main theorem). For all $m \geq 2$, the inequality

$$
-v_{2}\left(a_{2, m}\right) \leq v_{2}((2 m-2) !)
$$

holds. Equality holds if and only if $m$ is odd. Furthermore let $n$ be a positive integer so that $2^{n} \leq m \leq 2^{n+1}-1$. If $m$ is even and $m>2^{n+2-v_{2}(m)}-2 n+3 v_{2}(m)-3$,

$$
-v_{2}\left(a_{2, m}\right) \leq v_{2}(m)+v_{2}((m-1) !)+\sum_{k=1}^{n+1-v_{2}(m)} 2^{k}\left(n-k+1-v_{2}(m)\right)
$$

holds.

Remark 1.3. We note that the inequality

$$
v_{2}((2 m-2) !) \leq 2 m-3
$$

holds for all $m$. Further if $m$ even and $m>2^{n+2-v_{2}(m)}-2 n+3 v_{2}(m)-3$, then

$$
v_{2}(m)+v_{2}((m-1) !)+\sum_{k=1}^{n+1-v_{2}(m)} d^{k}\left(n-k+1-v_{2}(m)\right)<v_{2}((2 m-2) !)
$$

(see the proof of the main theorem for details and Tables 5, 6, 7).

Remark 1.4. The condition $m$ is even and $m>2^{n+2-v_{2}(m)}-2 n+3 v_{2}(m)-3$ is not empty. At least if $n \geq 2 v_{2}(m)$ and $v_{2}(m)>1$, then

$$
\begin{aligned}
& 2^{n+2}-v_{2}(m)-2 n+3 v_{2}(m)-3 \\
& \quad=1-v_{2}(m)+\left(2 v_{2}(m)-n\right) 2+2^{2}+2^{3}+\cdots+2^{n+1-v_{2}(m)} \\
& \quad<1+2+2^{2}+\cdots+2^{n+1-v_{2}(m)} \\
& \quad \leq 1+2+2^{2}+\cdots+2^{n-1} \\
& \quad<m
\end{aligned}
$$

since $2^{n} \leq m \leq 2^{n+1}-1$.

\section{Preliminaries}

We will use the following lemmas.

Lemma 2.1 ([7, pp. 69-72, and pp. 102-114]). Let $x, y \in \mathbb{R}$ and $p$ be a prime number. The following relations are valid:

$$
\begin{aligned}
& \lfloor x\rfloor+m=\lfloor x+m\rfloor \text { for } m \in \mathbb{Z} . \\
& \lfloor x\rfloor+\lfloor y\rfloor \leq\lfloor x+y\rfloor . \\
& \left\lfloor\frac{\lfloor x\rfloor}{m}\right\rfloor=\left\lfloor\frac{x}{m}\right\rfloor \text { for } m \in \mathbb{N} . \\
& v_{p}(m n)=v_{p}(m)+v_{p}(n) \text { for } m, n \in \mathbb{Z} . \\
& v_{p}(m / n)=v_{p}(m)-v_{p}(n) \text { for } m \in \mathbb{Z}, n \in \mathbb{Z} \backslash\{0\} . \\
& v_{p}(m+n) \geq \min \left\{v_{p}(m), v_{p}(n)\right\} \text { for } m, n \in \mathbb{Z} .
\end{aligned}
$$




$$
v_{p}(m !)=\sum_{l=1}^{\infty}\left\lfloor\frac{m}{p^{l}}\right\rfloor \text { for } m \in \mathbb{N}_{0} .
$$

Further the following Lemmas will be used in the proof.

Lemma 2.2 ([13, Theorem 6]). For $n \in \mathbb{N}$ and fixed $d \in \mathbb{N} \backslash\{1\}$, let $2 \leq m \leq d^{n+1}-1$ and $r$ sufficiently large. Then

$$
-m a_{d, m}=\frac{1}{2 \pi i} \int_{|w|=r} P_{d, w}^{\circ n}(w)^{m / d^{n}} \frac{\mathrm{d} w}{w^{2}} .
$$

Lemma 2.3 ([13, Corollary 8]). Let $n \in \mathbb{N}$ and $2 \leq m \leq d^{n+1}-1$. Then

$$
\begin{aligned}
m a_{d, m}= & \sum C_{j_{1}}\left(\frac{m}{d^{n}}\right) C_{j_{2}}\left(\frac{m}{d^{n-1}}-d j_{1}\right) C_{j_{3}}\left(\frac{m}{d^{n-2}}-d^{2} j_{1}-d j_{2}\right) \\
& \cdots C_{j_{n}}\left(\frac{m}{d}-d^{n-1} j_{1}-d^{n-2} j_{2}-\cdots-d j_{n-1}\right),
\end{aligned}
$$

where the sum is over all non-negative indices $j_{1}, \ldots, j_{n}$ such that $\left(d^{n}-1\right) j_{1}+\left(d^{n-1}-1\right) j_{2}+\left(d^{n-2}-1\right) j_{3}+\cdots+$ $(d-1) j_{n}=m-1$.

$C_{j}(a)$ in Lemma 2.3 is the general binomial coefficient, i.e.

$$
C_{j}(a)=\frac{a(a-1)(a-2) \cdots(a-j+1)}{j(j-1)(j-2) \cdots(1)}
$$

for any $a \in \mathbb{R}$ and $j \in \mathbb{N}$ with $C_{0}(a)=1$.

\section{Results}

We can now state our main result.

Theorem 3.1. For fixed $d \in \mathbb{N} \backslash\{1\}$, let $m \in \mathbb{N} \backslash\{1\}$ with $(d-1) \mid(m-1)$. We assume that $d$ is factorized as $d=p_{1}^{t_{1}} p_{2}^{t_{2}} \cdots p_{s}^{t_{s}}$ where $s \in \mathbb{N}, t_{1}, t_{2}, \ldots, t_{s} \in \mathbb{N}$ and $p_{1}, p_{2}, \ldots, p_{s}$ are distinct prime numbers. Let $A=(m-1) /(d-1)$. Then,

$$
-v_{p_{l}}\left(a_{d, m}\right) \leq v_{p_{l}}(A !)+t_{l} A
$$

holds for any $p_{l} \in\left\{p_{1}, p_{2}, \ldots, p_{s}\right\}$. Equality holds if and only if $p_{l} \nmid m$ and $m=d$. Furthermore let $n \in \mathbb{N}$ so that $d^{n} \leq m \leq d^{n+1}-1$ and set $N=n+1-v_{p_{l}}(m) / t_{l}$. If $p_{l} \mid m$, then the inequality

$$
-v_{p_{l}}\left(a_{d, m}\right) \leq v_{p_{l}}(m)+v_{p_{l}}(A !)+\sum_{k=1}^{\max \{\lfloor N\rfloor, 0\}} d^{k}\left(t_{l}(n-k+1)-v_{p_{l}}(m)\right)
$$

holds when either the two inequalities $n \geq v_{p_{l}}(m) / t_{l}$ and $m>1-v_{p_{l}}(m) / t_{l}+\left(2 v_{p_{l}}(m) / t_{l}-n\right) d+d^{2}+d^{3}+\cdots+$ $d^{\lfloor N\rfloor}+(N-\lfloor N\rfloor) d^{\lfloor N\rfloor+1}$ are true, or $n<v_{p_{l}}(m) / t_{l}$ is satisfied.

Remark 3.2. If $(d-1) \nmid(m-1)$, then $a_{d, m}=0$ by [13, Corollary 11] and hence $-v_{p_{l}}\left(a_{d, m}\right)=-\infty$ for any $p_{l} \in\left\{p_{1}, p_{2}, \ldots, p_{s}\right\}$ (the converse is not true, i.e. there are some zero-coefficients in the case $(d-1) \mid(m-1)($ see [13, Theorem 10 and Theorem 12]).)

Remark 3.3. If $p_{l} \mid m$, then the inequality

$$
v_{p_{l}}(m)+v_{p_{l}}(A !)+\sum_{k=1}^{\left\lfloor n+1-v_{p_{l}}(m) / t_{l}\right\rfloor} d^{k}\left(t_{l}(n-k+1)-v_{p_{l}}(m)\right)<v_{p_{l}}(A !)+t_{l} A
$$

holds when the two inequalities $n \geq v_{p_{l}}(m) / t_{l}$ and $m>1-v_{p_{l}}(m) / t_{l}+\left(2 v_{p_{l}}(m) / t_{l}-n\right) d+d^{2}+d^{3}+\cdots+d^{\lfloor N\rfloor}+$ $(N-\lfloor N\rfloor) d^{\lfloor N\rfloor+1}$ are true, or $n<v_{p_{l}}(m) / t_{l}$ is satisfied (see the proof for details).

Remark 3.4. The case $p_{l} \mid m, n \geq v_{p_{l}}(m) / t_{l}$ and $m>1-v_{p_{l}}(m) / t_{l}+\left(2 v_{p_{l}}(m) / t_{l}-n\right) d+d^{2}+d^{3}+\cdots+d^{\lfloor N\rfloor}+$ $(N-\lfloor N\rfloor) d^{\lfloor N\rfloor+1}$ is not empty. At least if $n \geq 2 v_{p_{l}}(m) / t_{l}$ and $v_{p_{l}}(m) / t_{l} \in \mathbb{N} \backslash\{1\}$, then

$$
\begin{aligned}
1- & v_{p_{l}}(m) / t_{l}+\left(2 v_{p_{l}}(m) / t_{l}-n\right) d+d^{2}+d^{3}+\cdots+d^{\lfloor N\rfloor}+(N-\lfloor N\rfloor) d^{\lfloor N\rfloor+1} \\
& =1-v_{p_{l}}(m) / t_{l}+\left(2 v_{p_{l}}(m) / t_{l}-n\right) d+d^{2}+d^{3}+\cdots+d^{\lfloor N\rfloor} \\
& <1+d+d^{2}+\cdots+d^{\lfloor N\rfloor} \\
& \leq 1+d+d^{2}+\cdots+d^{n-1}
\end{aligned}
$$




$$
\begin{aligned}
& =\left(d^{n}-1\right) /(d-1) \\
& <m
\end{aligned}
$$

because $d^{n} \leq m \leq d^{n+1}-1$ and $d=p_{1}^{t_{1}} p_{2}^{t_{2}} \cdots p_{s}^{t_{s}}$.

Using the main theorem, we obtain the following corollary for the growth of the denominator.

Corollary 3.5. Under the same assumption of the main theorem, $a_{d, m} d^{x(m)} \in \mathbb{Z}$ holds where

$$
x(m):=\max _{1 \leq j \leq s}\left\lceil\frac{v_{p_{j}}(A)+t_{j} A}{t_{j}}\right\rceil .
$$

The strategy is basically same as in the proof of [12]. However we need a more detailed calculation in the last part.

Proof of Theorem 3.1. We take $d \in \mathbb{N} \backslash\{1\}, m \in \mathbb{N} \backslash\{1\}$ with $(d-1) \mid(m-1)$. Assume that $d$ is factorized as $d=$ $p_{1}^{t_{1}} p_{2}^{t_{2}} \cdots p_{s}^{t_{s}}$ where $s \in \mathbb{N}, t_{1}, t_{2}, \ldots, t_{s} \in \mathbb{N}, p_{1}, p_{2}, \ldots, p_{s}$ are distinct prime numbers and set

$$
\begin{aligned}
\mathbb{J}= & \left\{\left(j_{1}, j_{2}, \ldots, j_{n}\right) \in\left(\mathbb{N}_{0}\right)^{n}:\right. \\
& \left.\left(d^{n}-1\right) j_{1}+\left(d^{n-1}-1\right) j_{2}+\cdots+(d-1) j_{n}=m-1\right\} .
\end{aligned}
$$

By Lemma 2.2 if $m=d^{n}$ for some $n \in \mathbb{N}$, then we have

$$
\begin{aligned}
d^{n} a_{d, d^{n}} & =\frac{1}{2 \pi i} \int_{|z|=r} P_{d, w}^{\circ n}(w)^{d^{n} / d^{n}} \frac{d w}{w^{2}} \\
& =\frac{1}{2 \pi i} \int_{|z|=r}\left(\left(P_{d, w}^{\circ n-1}(w)\right)^{d}+w\right) \frac{d w}{w^{2}} \\
& =1 .
\end{aligned}
$$

Hence $a_{d, d^{n}}=1 / d^{n}$. In particular $a_{d, d}=1 / d$. It means that $-v_{p_{l}}\left(a_{d, d}\right)=t_{l}$ holds when $m=d$.

Take $n \in \mathbb{N}$ such that $d^{n} \leq m \leq d^{n+1}-1$. Let $k \in\{1,2, \ldots, n\},\left(j_{1}, j_{2}, \ldots, j_{n}\right) \in \mathbb{J}$ and set

$$
\alpha_{k}=m / d^{n-k+1}-d^{k-1} j_{1}-d^{k-2} j_{2}-\cdots-d j_{k-1} .
$$

Setting $\beta=d^{n-k+1} \alpha_{k}=m-d^{n} j_{1}-d^{n-1} j_{2}-\cdots-d^{n-k+2} j_{k-1}$, we obtain

$$
\begin{aligned}
C_{j_{k}}\left(\alpha_{k}\right) & =\frac{\alpha_{k}\left(\alpha_{k}-1\right)\left(\alpha_{k}-2\right) \cdots\left(\alpha_{k}-\left(j_{k}-1\right)\right)}{j_{k} !} \\
& =\frac{\beta\left(\beta-d^{n-k+1}\right)\left(\beta-2 d^{n-k+1}\right) \cdots\left(\beta-\left(j_{k}-1\right) d^{n-k+1}\right)}{d^{j_{k}(n-k+1)} j_{k} !} .
\end{aligned}
$$

First we give an estimate for $-v_{p_{l}}\left(C_{j_{k}}\left(\alpha_{k}\right)\right)$ for fixed $l \in\{1,2, \ldots, s\}$ (the calculation is similar to [12]). The denominator of (3.3) satisfies the following equality:

$$
v_{p_{l}}\left(d^{j_{k}(n-k+1)} j_{k} !\right) \stackrel{(2.4)}{=} v_{p_{l}}\left(j_{k} !\right)+j_{k} t_{l}(n-k+1) .
$$

Furthermore we have

$$
\begin{aligned}
& v_{p_{l}}\left(\beta-(l-1) d^{n-k+1}\right) \\
& \quad=v_{p_{l}}\left(m-d^{n} j_{1}-d^{n-1} j_{2}-\cdots-d^{n-k+2} j_{k-1}-(l-1) d^{n-k+1}\right) \\
& \quad=v_{p_{l}}\left(m-d^{n-k+1}\left(d^{k-1} j_{1}-d^{k-2} j_{2}-\cdots-d j_{k-1}-(l-1)\right)\right) \\
& \quad \stackrel{(2.6)}{\geq} \min \left\{v_{p_{l}}(m), t_{l}(n-k+1)\right\}
\end{aligned}
$$

for all $l \in\left\{1,2, \ldots, j_{k}\right\}$. Hence the numerator of (3.3) satisfies the following inequality:

$$
\begin{aligned}
& v_{p_{l}}\left(\beta\left(\beta-d^{n-k+1}\right)\left(\beta-2 d^{n-k+1}\right) \cdots\left(\beta-\left(j_{k}-1\right) d^{n-k+1}\right)\right) \\
& \quad \stackrel{(2.4)}{\geq} j_{k} \min \left\{v_{p_{l}}(m), t_{l}(n-k+1)\right\} .
\end{aligned}
$$

Combining (3.4) and (3.5), we obtain

$$
\begin{aligned}
-v_{p_{l}}\left(C_{j_{k}}\left(\alpha_{k}\right)\right) \stackrel{(2.5)}{\leq} v_{p_{l}}\left(j_{k} !\right)+j_{k} t_{l}(n-k+1)-j_{k} \min \left\{v_{p_{l}}(m), t_{l}(n-k+1)\right\} \\
=v_{p_{l}}\left(j_{k} !\right)+j_{k} \max \left\{t_{l}(n-k+1)-v_{p_{l}}(m), 0\right\} .
\end{aligned}
$$

Due to Lemma 2.3, we have 


$$
\begin{aligned}
-v_{p_{l}}\left(a_{d, m}\right) \leq & v_{p_{l}}(m)+\max _{\left(j_{1}, j_{2}, \ldots, j_{n}\right) \in \mathbb{J}}\left\{\sum_{k=1}^{n} v_{p_{l}}\left(j_{k} !\right)\right\} \\
& +\max _{\left(j_{1}, j_{2}, \ldots, j_{n}\right) \in \mathbb{J}}\left\{\sum_{k=1}^{n} j_{k} \max \left\{t_{l}(n-k+1)-v_{p_{l}}(m), 0\right\}\right\} .
\end{aligned}
$$

Considering the equation

$$
\left(d^{n}-1\right) j_{1}+\left(d^{n-1}-1\right) j_{2}+\cdots+(d-1) j_{n}=m-1
$$

for all $\left(j_{1}, j_{2}, \ldots, j_{n}\right) \in \mathbb{J}$ and $(2.2)$, we obtain

$$
\begin{aligned}
\sum_{k=1}^{n} v_{p_{l}}\left(j_{k} !\right) & \stackrel{(2.7)}{=} \sum_{k=1}^{n} \sum_{h=1}^{\infty}\left\lfloor\frac{j_{k}}{p_{l}^{h}}\right\rfloor \\
& \stackrel{(2.2)}{\leq} \sum_{h=1}^{\infty}\left\lfloor\frac{j_{1}+\cdots+j_{n}}{p_{l}^{h}}\right\rfloor \\
& \stackrel{(3.7)}{\leq} \sum_{h=1}^{\infty}\left\lfloor\frac{m-1}{(d-1) p_{l}^{h}}\right\rfloor \\
& \stackrel{(2.7)}{=} v_{p_{l}}(A !)
\end{aligned}
$$

for all $1 \leq k \leq n$. We immediately see that equality holds when $j_{1}=j_{2}=\cdots=j_{n-1}=0, j_{n}=(m-1) /(d-1)=A$. Conversely if equality holds, then actually $j_{1}=j_{2}=\cdots=j_{n-1}=0, j_{n}=A$, because $\left(j_{1}, j_{2}, \ldots, j_{n}\right) \in \mathbb{J}$ satisfies

$$
\left(d^{n-1}+d^{n-2}+\cdots+1\right) j_{1}+\cdots+(d+1) j_{n-1}+j_{n}=\frac{m-1}{d-1}=A
$$

and hence

$$
j_{1}+\cdots+j_{n}=A
$$

is valid only when $j_{1}=j_{2}=\cdots=j_{n-1}=0, j_{n}=A$.

If $p_{l} \nmid m$,

$$
\begin{aligned}
& \sum_{k=1}^{n} j_{k} \max \left\{t_{l}(n-k+1)-v_{p_{l}}(m), 0\right\} \\
& \quad=\sum_{k=1}^{n} j_{k} t_{l}(n-k+1) \\
& \quad \leq \sum_{k=1}^{n} j_{k} t_{l} \frac{d^{n-k+1}-1}{d-1} \\
& \quad \stackrel{(3.7)}{=} t_{l} A
\end{aligned}
$$

holds for all $\left(j_{1}, j_{2}, \ldots, j_{n}\right) \in \mathbb{J}$, because $v_{p_{l}}(m)=0$ for $p_{l} \nmid m$. Equality holds if and only if $j_{1}=j_{2}=\cdots=$ $j_{n-1}=0, j_{n}=A$. We note that if $j_{1}=j_{2}=\cdots=j_{n-1}=0, j_{n}=A$, then

$$
C_{0}\left(m / d^{n}\right) \cdot C_{0}\left(m / d^{n-1}\right) \cdots C_{A}(m / d)=C_{A}(m / d) \neq 0 .
$$

Considering the elements of the sum in the formula in Lemma 2.3, we have $a_{d, m} \neq 0$ and precisely $-v_{p_{l}}\left(a_{d, m}\right)=$ $v_{p_{l}}(A !)+t_{l} A$ if $p_{l} \backslash X_{m}$.

Furthermore we consider the remainder case of the above, i.e., the case $p_{l} \mid m$. If $n t_{l}>v_{p_{l}}(m)$, there exists $\left(j_{1}, j_{2}, \ldots, j_{n}\right) \in \mathbb{J}$ such that

$$
\begin{aligned}
& \max _{\left(j_{1}, j_{2}, \ldots, j_{n}\right) \in \mathbb{J}}\left\{\sum_{k=1}^{n} j_{k} \max \left\{t_{l}(n-k+1)-v_{p_{l}}(m), 0\right\}\right\} \\
& =\sum_{k=1}^{\left\lfloor n+1-v_{p_{l}}(m) / t_{l}\right\rfloor} j_{k}\left(t_{l}(n-k+1)-v_{p_{l}}(m)\right) \\
& =\sum_{k=1}^{\left\lfloor n+1-v_{p_{l}}(m) / t_{l}\right\rfloor} j_{k} t_{l}(n-k+1)-\sum_{k=1}^{\left\lfloor n+1-v_{p_{l}}(m) / t_{l}\right\rfloor} j_{k} v_{p_{l}}(m) .
\end{aligned}
$$

We see that 


$$
\begin{aligned}
\sum_{k=1}^{n^{\prime}} j_{k} t_{l}(n-k+1) & \leq \sum_{k=1}^{n} j_{k} t_{l}(n-k+1) \\
& \leq \max _{\left(j_{1}, j_{2}, \ldots, j_{n}\right) \in \mathbb{J}}\left\{\sum_{k=1}^{n} j_{k} t_{l} \frac{d^{n-k+1}-1}{d-1}\right\} \\
& =t_{l} A .
\end{aligned}
$$

Equality holds if and only if $j_{1}=j_{2}=\cdots=j_{n-1}=0, j_{n}=A$. On the other hand,

$$
\sum_{k=1}^{n^{\prime}} j_{k} v_{p_{l}}(m) \geq v_{p_{l}}(m) \text {. }
$$

In this case equality holds if and only if $j_{n}=\cdots=j_{n-1}=0, j_{n}=1$. Hence,

$$
\begin{aligned}
& \max _{\left(j_{1}, j_{2}, \ldots, j_{n}\right) \in \mathbb{J}}\left\{\sum_{k=1}^{n} j_{k} \max \left\{t_{l}(n-k+1)-v_{p_{l}}(m), 0\right\}\right\} \\
& <t_{l} A-v_{p_{l}}(m) .
\end{aligned}
$$

Therefore, if $p_{l} \mid m$ and $n t_{l}>v_{p_{l}}(m)$,

$$
\begin{aligned}
-v_{p_{l}}\left(a_{d, m}\right) & <v_{p_{l}}(m)+v_{p_{l}}(A !)+t_{l} A-v_{p_{l}}(m) \\
& =v_{p_{l}}(A !)+t_{l} A .
\end{aligned}
$$

Further if $m>1-v_{p_{l}}(m) / t_{l}+\left(2 v_{p_{l}}(m) / t_{l}-n\right) d+d^{2}+d^{3}+\cdots+d^{\lfloor N\rfloor}+(N-\lfloor N\rfloor) d^{\lfloor N\rfloor+1}$, or $n<v_{p_{l}}(m) / t_{l}$,

$$
\begin{aligned}
& \max _{\left(j_{1}, j_{2}, \ldots, j_{n}\right) \in \mathbb{J}}\left\{\sum_{k=1}^{n} j_{k} \max \left\{t_{l}(n-k+1)-v_{p_{l}}(m), 0\right\}\right\} \\
& =\sum_{k=1}^{\left\lfloor n+1-v_{p_{l}}(m) / t_{l}\right\rfloor} j_{k}\left(t_{l}(n-k+1)-v_{p_{l}}(m)\right) \\
& \leq \sum_{k=1}^{\left\lfloor n+1-v_{p_{l}}(m) / t_{l}\right\rfloor} d^{k}\left(t_{l}(n-k+1)-v_{p_{l}}(m)\right) \\
& <t_{l} A-v_{p_{l}}(m)
\end{aligned}
$$

and hence

$$
-v_{p_{l}}\left(a_{d, m}\right) \leq v_{p_{l}}(m)+v_{p_{l}}((m-1) !)+\sum_{k=1}^{\left\lfloor n+1-v_{p_{l}}(m) / t_{l}\right\rfloor} d^{k}\left(t_{l}(n-k+1)-v_{p_{l}}(m)\right) .
$$

On the other hand if $n t_{l} \leq v_{p_{l}}(m)$,

$$
\max _{\left(j_{1}, j_{2}, \ldots, j_{n}\right) \in \mathbb{J}}\left\{\sum_{k=1}^{n} j_{k} \max \left\{t_{l}(n-k+1)-v_{p_{l}}(m), 0\right\}\right\}=0
$$

and hence $-v_{p_{l}}\left(a_{d, m}\right) \leq v_{p_{l}}(m)+v_{p_{l}}(A !)<t_{l} A+v_{p_{l}}(A !)$.

Remark 3.6. The straightforward adaptation of this proof slightly improves the main result of [12] as follows:

Theorem 3.7 (Improved version of the main theorem of [12]). For fixed $d \in \mathbb{N} \backslash\{1\}$, let $m \in \mathbb{N}_{0}$ with $(d-1) \mid(m+1)$. We assume that $d$ is factorized as $d=p_{1}^{t_{1}} p_{2}^{t_{2}} \ldots p_{s}^{t_{s}}$ where $s \in \mathbb{N}, t_{1}, t_{2}, \ldots, t_{s} \in \mathbb{N}$ and $p_{1}, p_{2}, \ldots, p_{s}$ are distinct prime numbers. Let $B=(m+1) /(d-1)$. Then,

$$
-v_{p_{l}}\left(b_{d, m}\right) \leq v_{p_{l}}(B !)+t_{l} B
$$

holds for any $p_{l} \in\left\{p_{1}, p_{2}, \ldots, p_{s}\right\}$. Equality holds if and only if $p_{l} \nmid m$ and $m=d-2$. Furthermore let $n \in \mathbb{N}$ so that $d^{n}-2 \leq m \leq d^{n+1}-3$ and set $N=n+1-v_{p_{l}}(m) / t_{l}$. If $p_{l} \mid m$, the inequality

$$
-v_{p_{l}}\left(a_{d, m}\right) \leq v_{p_{l}}(m)+v_{p_{l}}(B !)+\sum_{k=1}^{\max \{\lfloor N\rfloor, 0\}} d^{k}\left(t_{l}(n-k+1)-v_{p_{l}}(m)\right)
$$

holds when either the two inequalities $n \geq v_{p_{l}}(m) / t_{l}$ and $m>-1-v_{p_{l}}(m) / t_{l}+\left(2 v_{p_{l}}(m) / t_{l}-n\right) d+d^{2}+d^{3}+\cdots+$ $d^{\lfloor N\rfloor}+(N-\lfloor N\rfloor) d^{\lfloor N\rfloor+1}$ are true, or $n<v_{p_{l}}(m) / t_{l}$ is satisfied.

In particular, the case $d=2$ is obtained as follows: 
Corollary 3.8. For all $m \geq 0$, the inequality

$$
-v_{2}\left(b_{2, m}\right) \leq v_{2}((2 m+2) !)
$$

holds. Equality holds if and only if $m$ is odd. Furthermore let $n \in \mathbb{N}$ so that $2^{n}-2 \leq m \leq 2^{n+1}-3$. If $m$ is even and $m>2^{n+2-v_{2}(m)}-2 n+3 v_{2}(m)-5$, then

$$
-v_{2}\left(b_{2, m}\right) \leq v_{2}(m)+v_{2}((m+1) !)+\sum_{k=1}^{n+1-v_{2}(m)} 2^{k}\left(n-k+1-v_{2}(m)\right) .
$$

\section{Tables}

We show some tables of coefficients $a_{d, m}$ (Tables 1, 2, 3 and 4) and our estimates (Tables 5, 6 and 7). We see that Theorem 3.7 give a better estimate of the main theorem of [12]. However, our estimates are not sharp as Tables 5, 6 and 7.

\begin{tabular}{|c|c|c|c|}
\hline $\mathrm{m}$ & Numerator & Denominator & $-v_{2}\left(a_{2, m}\right)$ \\
\hline 2 & 1 & 2 & 1 \\
\hline 3 & 1 & 8 & 3 \\
\hline 4 & 1 & 4 & 2 \\
\hline 5 & 15 & 128 & 7 \\
\hline 6 & 0 & - & $-\infty$ \\
\hline 7 & 81 & 1024 & 10 \\
\hline 8 & 1 & 8 & 3 \\
\hline 9 & 1499 & 32768 & 15 \\
\hline 10 & 1 & 32 & 5 \\
\hline 11 & 16551 & 262144 & 18 \\
\hline 12 & 0 & - & $-\infty$ \\
\hline 13 & -19557 & 4194304 & 22 \\
\hline 14 & 7 & 256 & 8 \\
\hline 15 & 1026129 & 33554432 & 25 \\
\hline 16 & 1 & 16 & 4 \\
\hline 17 & 78558483 & 2147483648 & 31 \\
\hline 18 & 7 & 512 & 9 \\
\hline 19 & 496067595 & 17179869184 & 34 \\
\hline 20 & 0 & - & $-\infty$ \\
\hline 21 & -506111055 & 274877906944 & 38 \\
\hline 22 & 135 & 4096 & 12 \\
\hline 23 & 66414150615 & 2199023255552 & 41 \\
\hline 24 & 0 & - & $-\infty$ \\
\hline 25 & 402782136143 & 70368744177664 & 46 \\
\hline 26 & 683 & 65536 & 16 \\
\hline 27 & -7661205650557 & 562949953421312 & 49 \\
\hline 28 & 0 & - & $-\infty$ \\
\hline 29 & 159606082621811 & 9007199254740992 & 53 \\
\hline 30 & 159 & 16384 & 14 \\
\hline
\end{tabular}

Table 1. The values of $a_{2, m}$. 
Table 2. The values of $a_{3, m}$.

\begin{tabular}{|c|c|c|c|}
\hline $\mathrm{m}$ & Numerator & Denominator & $-v_{3}\left(a_{3, m}\right)$ \\
\hline 3 & 1 & 3 & 1 \\
\hline 5 & 1 & 9 & 2 \\
\hline 7 & 2 & 81 & 4 \\
\hline 9 & 1 & 9 & 2 \\
\hline 11 & 52 & 729 & 6 \\
\hline 13 & 155 & 6561 & 8 \\
\hline 15 & 0 & - & $-\infty$ \\
\hline 17 & 2657 & 59049 & 10 \\
\hline 19 & 29533 & 1594323 & 13 \\
\hline 21 & 0 & - & $-\infty$ \\
\hline 23 & -69655 & 14348907 & 15 \\
\hline 25 & 2969930 & 129140163 & 17 \\
\hline 27 & 1 & 27 & 3 \\
\hline 29 & 23095973 & 1162261467 & 19 \\
\hline 31 & 56696777 & 10460353203 & 21 \\
\hline 33 & 10 & 729 & 6 \\
\hline 35 & 2343898963 & 94143178827 & 23 \\
\hline 37 & 24995524274 & 2541865828329 & 26 \\
\hline 39 & 0 & - & $-\infty$ \\
\hline 41 & 115000492832 & 22876792454961 & 28 \\
\hline 43 & 3201040250650 & 205891132094649 & 30 \\
\hline 45 & 0 & - & $-\infty$ \\
\hline 47 & -6747874422283 & 1853020188851841 & 32 \\
\hline 49 & 27156979500091 & 16677181699666569 & 34 \\
\hline
\end{tabular}

Table 3. The values of $a_{4, m}$.

\begin{tabular}{cccc}
\hline $\mathrm{m}$ & Numerator & Denominator & $-v_{2}\left(a_{4, m}\right)$ \\
\hline 4 & 1 & 4 & 2 \\
7 & 3 & 32 & 5 \\
10 & 1 & 32 & 5 \\
13 & 15 & 2048 & 11 \\
16 & 1 & 16 & 4 \\
19 & 2995 & 65536 & 16 \\
22 & 93 & 4096 & 23 \\
25 & 59451 & 83886608 & $-\infty$ \\
28 & 0 & - & 28 \\
31 & 7405653 & 2684355456 & 20 \\
34 & 17127 & 1048576 & 34 \\
37 & 102177851 & 171798699184 & 39 \\
43 & -1017988077 & 549755813888 & 27 \\
46 & 2092125 & 134217728 & 47 \\
49 & 716781072211 & 1407374883555328 & $-\infty$ \\
52 & 0 & - & 52 \\
55 & -8057836991135 & 4503599627370496 & 36 \\
58 & -107583317 & 68719476736 & 58 \\
61 & 2910453741726705 & 288230376151711744 & 6 \\
64 & 1 & 64 & 63 \\
67 & 91893393031048069 & 92233720368554775808 & 43 \\
70 & 37808167947 & 8796093022208 & 70 \\
73 & 1318087272305007215 & 1180591620717411303424 & 75 \\
76 & 231 & 32768 & \\
79 & 444913124772728735913 & 37778931862957161709568 & \\
\hline
\end{tabular}


Table 4. The values of $a_{6, m}$.

\begin{tabular}{cccc}
\hline $\mathrm{m}$ & Numerator & Denominator & $-v_{2}\left(a_{6, m}\right)$ \\
\hline 6 & 1 & 6 & 1 \\
11 & 5 & 72 & 3 \\
16 & 5 & 162 & 1 \\
21 & 5 & 384 & 7 \\
26 & 7 & 1458 & 1 \\
31 & 8645 & 6718464 & 10 \\
36 & 1 & 36 & 2 \\
41 & 44166115 & 1934917632 & 15 \\
46 & 96545 & 6377292 & 2 \\
51 & 20051 & 2359296 & 18 \\
56 & 224695 & 57395628 & 2 \\
61 & 682050153785 & 541653102231552 & 22 \\
66 & 0 & - & $-\infty$ \\
71 & 510189065505655 & 38999023360671744 & 25 \\
76 & 412426453 & 41841412812 & 2 \\
81 & 120083275 & 19327352832 & 31 \\
86 & 2394396445 & 753145430616 & 3 \\
91 & 49144739612524327415 & 43668922413972021313536 & 34 \\
96 & 0 & - & $-\infty$ \\
101 & -2801171227435232984071 & 6288324827611971069149184 & 38 \\
106 & 52774878534565 & 6588516227028768 & 5 \\
111 & 2597391412505 & 534362651099136 & 46 \\
116 & 19211930633005 & 7412080755407364 & \\
\hline 121 & 1137781778131315301990813815 & & \\
& & 1173552332628256488808897314816 & \\
\end{tabular}

Table 5. The comparison of of the estimates for $d=2$.

\begin{tabular}{|c|c|c|c|c|}
\hline$m$ & $-v_{2}\left(a_{2, m}\right)$ & Theorem A (1.1) & Corollary 1.1 (1.2) & Corollary $1.1(1.3)$ \\
\hline 2 & 1 & 1 & 1 & - \\
\hline 3 & 3 & 3 & 3 & - \\
\hline 4 & 2 & 5 & 4 & 3 \\
\hline 5 & 7 & 7 & 7 & - \\
\hline 6 & $-\infty$ & 9 & 8 & 6 \\
\hline 7 & 10 & 11 & 10 & - \\
\hline 8 & 3 & 13 & 11 & 7 \\
\hline 9 & 15 & 15 & 15 & - \\
\hline 10 & 5 & 17 & 16 & - \\
\hline 11 & 18 & 19 & 18 & - \\
\hline 12 & $-\infty$ & 21 & 19 & 12 \\
\hline 13 & 22 & 23 & 22 & - \\
\hline 14 & 8 & 25 & 23 & 19 \\
\hline 15 & 25 & 27 & 25 & - \\
\hline 16 & 4 & 29 & 26 & 15 \\
\hline 17 & 31 & 31 & 31 & - \\
\hline 18 & 9 & 33 & 32 & - \\
\hline 19 & 34 & 35 & 34 & - \\
\hline 20 & $-\infty$ & 37 & 35 & 26 \\
\hline 21 & 38 & 39 & 38 & - \\
\hline 22 & 12 & 41 & 39 & - \\
\hline 23 & 41 & 43 & 41 & - \\
\hline 24 & $-\infty$ & 45 & 42 & 24 \\
\hline 25 & 46 & 47 & 46 & - \\
\hline 26 & 16 & 49 & 47 & 45 \\
\hline 27 & 49 & 51 & 49 & - \\
\hline 28 & $-\infty$ & 53 & 50 & 33 \\
\hline 29 & 53 & 55 & 53 & - \\
\hline 30 & 14 & 57 & 54 & 48 \\
\hline
\end{tabular}


Table 6. The comparison of of the estimates for $d=4$ and $p=2$.

\begin{tabular}{|c|c|c|c|}
\hline$m$ & $-v_{2}\left(a_{4, m}\right)$ & Theorem 3.1 (3.1) & Theorem 3.1 (3.2) \\
\hline 4 & 2 & 2 & - \\
\hline 7 & 5 & 5 & - \\
\hline 10 & 5 & 7 & 6 \\
\hline 13 & 11 & 11 & - \\
\hline 16 & 4 & 13 & 7 \\
\hline 19 & 16 & 16 & - \\
\hline 22 & 12 & 18 & - \\
\hline 25 & 23 & 23 & - \\
\hline 28 & $-\infty$ & 25 & 17 \\
\hline 31 & 28 & 28 & - \\
\hline 34 & 20 & 30 & - \\
\hline 37 & 34 & 34 & - \\
\hline 40 & $-\infty$ & 36 & 17 \\
\hline 43 & 39 & 39 & - \\
\hline 46 & 27 & 41 & 40 \\
\hline 49 & 47 & 47 & - \\
\hline 52 & $-\infty$ & 49 & 25 \\
\hline 55 & 52 & 52 & - \\
\hline 58 & 36 & 54 & 45 \\
\hline 61 & 58 & 58 & - \\
\hline 64 & 6 & 60 & 24 \\
\hline 67 & 63 & 63 & - \\
\hline 70 & 43 & 65 & - \\
\hline 73 & 70 & 70 & - \\
\hline 76 & 15 & 72 & - \\
\hline 79 & 75 & 75 & - \\
\hline 82 & 51 & 77 & - \\
\hline 85 & 81 & 81 & - \\
\hline 88 & 0 & 83 & 56 \\
\hline 91 & 86 & 86 & - \\
\hline 94 & 58 & 88 & - \\
\hline 97 & 95 & 95 & - \\
\hline 100 & $-\infty$ & 97 & 81 \\
\hline 103 & 100 & 100 & - \\
\hline 106 & 68 & 102 & - \\
\hline 109 & 106 & 106 & - \\
\hline 112 & $-\infty$ & 108 & 46 \\
\hline 115 & 111 & 111 & - \\
\hline 118 & 75 & 113 & - \\
\hline 121 & 118 & 118 & - \\
\hline 124 & 25 & 120 & 88 \\
\hline 127 & 123 & 123 & - \\
\hline 130 & 83 & 125 & - \\
\hline 133 & 129 & 129 & - \\
\hline 136 & 19 & 131 & 72 \\
\hline 139 & 134 & 134 & - \\
\hline 142 & 90 & 136 & - \\
\hline 145 & 142 & 142 & - \\
\hline 148 & $-\infty$ & 144 & 96 \\
\hline 151 & 147 & 147 & - \\
\hline 154 & 99 & 149 & - \\
\hline 157 & 153 & 153 & - \\
\hline 160 & $-\infty$ & 155 & 58 \\
\hline 163 & 158 & 158 & - \\
\hline 166 & 106 & 160 & - \\
\hline 169 & 165 & 165 & - \\
\hline 172 & 32 & 167 & 103 \\
\hline 175 & 170 & 170 & - \\
\hline
\end{tabular}


Table 7. The comparison of of the estimates for $d=6$ and $p=2$.

\begin{tabular}{|c|c|c|c|}
\hline$m$ & $-v_{2}\left(a_{6, m}\right)$ & Theorem 3.1 (3.1) & Theorem 3.1 (3.2) \\
\hline 6 & 1 & 1 & - \\
\hline 11 & 3 & 3 & - \\
\hline 16 & 1 & 4 & - \\
\hline 21 & 7 & 7 & - \\
\hline 26 & 1 & 8 & 4 \\
\hline 31 & 10 & 10 & - \\
\hline 36 & 2 & 11 & 6 \\
\hline 41 & 15 & 15 & - \\
\hline 46 & 2 & 16 & 14 \\
\hline 51 & 18 & 18 & - \\
\hline 56 & 2 & 19 & 11 \\
\hline 61 & 22 & 22 & - \\
\hline 66 & $-\infty$ & 23 & 17 \\
\hline 71 & 25 & 25 & - \\
\hline 76 & 2 & 26 & 13 \\
\hline 81 & 31 & 31 & - \\
\hline 86 & 3 & 32 & 22 \\
\hline 91 & 34 & 34 & - \\
\hline 96 & $-\infty$ & 35 & 21 \\
\hline 101 & 38 & 38 & - \\
\hline 106 & 5 & 39 & 25 \\
\hline 111 & 41 & 41 & - \\
\hline 116 & 2 & 42 & 21 \\
\hline 121 & 46 & 46 & - \\
\hline 126 & $-\infty$ & 47 & 29 \\
\hline 131 & 49 & 49 & - \\
\hline 136 & 1 & 50 & 26 \\
\hline 141 & 53 & 53 & - \\
\hline 146 & 8 & 54 & 32 \\
\hline 151 & 56 & 56 & - \\
\hline 156 & $-\infty$ & 57 & 28 \\
\hline 161 & 63 & 63 & - \\
\hline 166 & 7 & 64 & 38 \\
\hline 171 & 66 & 66 & - \\
\hline 176 & $-\infty$ & 67 & 36 \\
\hline 181 & 70 & 70 & - \\
\hline 186 & $-\infty$ & 71 & 41 \\
\hline 191 & 73 & 73 & - \\
\hline 196 & 2 & 74 & 37 \\
\hline 201 & 78 & 78 & - \\
\hline 206 & 9 & 79 & 45 \\
\hline 211 & 81 & 81 & - \\
\hline 216 & 3 & 82 & 42 \\
\hline 221 & 85 & 85 & - \\
\hline 226 & 11 & 86 & - \\
\hline 231 & 88 & 88 & - \\
\hline 236 & 3 & 89 & 50 \\
\hline 241 & 94 & 94 & - \\
\hline 246 & 12 & 95 & - \\
\hline 256 & 3 & 98 & 55 \\
\hline 261 & 101 & 101 & - \\
\hline 266 & 12 & 102 & 98 \\
\hline 271 & 104 & 104 & - \\
\hline 276 & $-\infty$ & 105 & 58 \\
\hline 281 & 109 & 109 & - \\
\hline 286 & 16 & 110 & 102 \\
\hline 291 & 112 & 112 & - \\
\hline
\end{tabular}




\section{Acknowledgement}

The author is grateful to Professor Toshiyuki Sugawa for his helpful suggestions and constant encouragement. He further expresses his deep gratitude to the referees for their careful reading of this manuscript and for their helpful comments.

\section{REFERENCES}

[1] Bielefeld, B., Fisher, Y., and Haeseler, F. V., "Computing the Laurent series of the map $\Psi: C \backslash \bar{D} \rightarrow C \backslash M$," Adv. Appl. Math., 14: 25-38 (1993).

[2] Douady, A., and Hubbard, J. H., Exploring the Mandelbrot Set, The Orsay Notes (1985).

[3] Douady, A., and Hubbard, J. H., "Itération des polynômes quadratiques complexes," C. R. Acad. Sci. Paris, 294: 123-126 (1982).

[4] Ewing, J., and Schober, G., "On the coefficients of the mapping to the exterior of the Mandelbrot set," Michigan Math. J., 37: 315-320 (1990).

[5] Ewing, J., and Schober, G., "The area of the Mandelbrot set," Numerische Mathematik, 61: 59-72 (1992).

[6] Ewing, J., and Schober, G., "Coefficients associated with the reciprocal of the Mandelbrot set," J. Math. Anal. Appl., 170(1): 104-114 (1992).

[7] Graham, R. L., Knuth, D. E., and Patashnik, O., Concrete Mathematics, Addison-Wesley (1988).

[8] Jungreis, I., "The uniformization of the complement of the Mandelbrot set," Duke Math. J., 52(4): $935-938$ (1985).

[9] Lau, E., and Schleicher, D., "Symmetries of fractals revisited," Math. Intelligencer, 18(1): 45-51 (1996).

[10] Levin, G. M., "On the arithmetic properties of a certain sequence of polynomials," Russian Math. Surveys, 43: 245-246 (1988).

[11] Morosawa, S., Nishimura, Y., Taniguchi, M., and Ueda, T., Holomorphic Dynamics, Cambridge Univ. Press, Cambridge (2000).

[12] Shimauchi, H., “A remark on Zagier's observation of the Mandelbrot set," Osaka J. Math., 52(3): 737-747.

[13] Shimauchi, H., On the Coefficients of the Riemann Mapping Function for the Exterior of the Multibrot Set, Topics in Finite or Infinite Dimensional Complex Analysis, 237-248, Tohoku University Press, Sendai (2013).

[14] Shimauchi, H., On the Coefficients of the Riemann Mapping Function for the Exterior of the Mandelbrot Set (in japanese), Master thesis (2012), Graduate School of Information Sciences, Tohoku University.

[15] Yamashita, O., On the Coefficients of the Mapping to the Exterior of the Mandelbrot Set (in japanese), Master thesis (1998), Graduate School of Information Science, Nagoya University, http://www.math.human.nagoya-u.ac.jp/master.thesis/1997.html. 